\title{
Nonlinear super-resolution signal processing for subcellular analysis of calcium dynamics
}

\author{
Niccolò Calcini ${ }^{1}$, æa, * , Angelica da Silva Lantyer ${ }^{1, ~ a b}$, Fleur Zeldenrust $^{1}$, Tansu \\ Celikel $^{1, \text { ac }}$, \\ 1 Department of Neurophysiology, Donders Institute for Brain, Cognition and \\ Behaviour, Radboud University, Heyedaalseweg 135, 6525 HJ, Nijmegen - The \\ Netherlands
}

Ca Current Address: Department for BioMedical Research (DMBR), University of Bern, Bern, Switzerland

ab Current Address: Department of Cognitive \& Systems Neuroscience (CSN) /

Swammerdam Institute for Life Science (SILS) / Faculty of Science, University of

Amsterdam, Amsterdam, The Netherlands

Oc Current Address: Georgia Institute of Technology, Atlanta, GA

*n.calcini@neurophysiology.nl

\begin{abstract}
Optical (fluorescence) imaging of ionic dynamics has revolutionized neuroscience as it allows the study of neural activity across spatially identified populations. Quantification of fluorescence signals is commonly performed using ratiometric measures, like the $\Delta \mathrm{F} / \mathrm{F}$. Although these measures are robust and easy to implement, they do not take advantage of the temporal information available in time-varying signals. Moreover, since a baseline (reference) period needs to be selected, their application is often limited to the quantification of stimulus-evoked activity. Here, we present a new approach, called ARES, based on the quantification of residuals after linear autoregression. We demonstrate the utility of ARES to quantify the functional dynamics of stimulus representation in cortical networks and show that it improves the spatial and temporal resolution with respect to $\Delta \mathrm{F} / \mathrm{F}$. We further show that ARES can be used to study subcellular calcium dynamics and exemplify its utility to describe the spatiotemporal dynamics of calcium signal localization in compartmental network recordings. ARES offers a novel method for quantitative analysis of optical imaging data
\end{abstract}

\section{Author summary}

Two-photon calcium imaging is a powerful and non-invasive method to measure and visualize the activity of neural populations in single cell and subcellular resolution. However, the slow dynamics of both the calcium signal itself and its indicators make it difficult to relate the timing and origin of the neural activity to the calcium signal. The most commonly used analysis method, $\Delta \mathrm{F} / \mathrm{F}$, uses a reference baseline to quantify signal ratiometrically. This results in several limitations, especially in accounting for the time varying fluctuations in fluorescence values. Here we present a new method for the analysis of calcium signals, called ARES, in which a sliding window is used to predict the future values of the signal and deviations from this prediction are recorded as the signal. This has the advantage that shifts in baseline do not affect the processing, and that the method has an improved spatial and temporal resolution with respect to $\Delta \mathrm{F} / \mathrm{F}$. 


\section{Introduction}

Neuronal activity is traditionally studied using electrophysiological methods.

Intracellular and extracellular electrodes provide the best selectivity (i.e. isolated single neurons) and highest temporal resolution, however, their application necessitates invasive interventions with neural circuits. Moreover, because electrophysiological methods provide only limited spatial information (e.g. cortical depth), their application to study spatially well-defined local network dynamics has been limited. Development of inorganic ion and voltage-sensitive dyes 1, 1,2 and subsequent advances in genetically engineered sensors, in particular, calcium indicators, now enable visualization of intact neuronal populations while providing functional insight into the dynamics of the network activity [3].

Notwithstanding its importance, the calcium imaging technique has several limitations. First, the nonlinear and slow dynamics of cellular calcium concentrations and indicators hamper the efforts to reconstruct the spiking activity of neurons from calcium traces [4.5]. Second, chromophores undergo "photobleaching", where the structure of the molecules themselves is modified making them permanently lose their light emission properties. Photobleaching is particularly prominent during prolonged recording sessions, which is common in functional studies and live recordings of neurons [6]. Finally, light scattering contributes to the decrease of the spatial signal to noise ratio and is often difficult to control.

The current standard analysis methods for calcium imaging recordings are ratiometric measures based on the difference between the signal (of interest) and a baseline [7], or between two different wavelengths as applied during Fluorescence Resonance Energy Transfer (FRET) imaging [8, 9]. $\Delta \mathrm{F} / \mathrm{F}$, which is the difference in fluorescence between any frame and a reference $(\Delta \mathrm{F})$, normalized by the reference value $(/ F)$, is by far the most common analysis method for functional calcium imaging. The simplicity and speed of implementation and the ease of interpretation of this ratiometric analysis method ensured its widespread implementation. However, like any other method, $\Delta \mathrm{F} / \mathrm{F}$ has its limitations. As it is based on the comparison with a baseline value, a static baseline can influence the detection or quantification of subsequent events in time-varying signals, commonly observed during prolonged recordings. $\Delta \mathrm{F} / \mathrm{F}$ also fails to correct for photobleaching effects in case of long time intervals between the current signal and the baseline value (i.e. prolonged recordings), as the dampening of the signal is included in the difference in fluorescence. As an attempt to overcome some of these shortcomings, and to take advantage of the time dimension present in functional recordings, we developed a new method for the analysis of functional calcium imaging data. This method, which we call ARES (for Autoregression RESiduals), is based on the analysis of a sliding time window of fixed length, to which we apply a pixel-wise linear autoregression (predicting a pixel value on the basis of its past values, see the Methods Section for detailed information about the computations involved). The time series of the residuals obtained by the comparison between the predicted signal and the measured signal is averaged over a time window to reduce the noise and to isolate the transient (novel) events in the signal. Application of ARES to the analysis of network dynamics during single cell stimulation showed that ARES improves the spatial and temporal resolution in functional signals respects to the raw signal and standard $\Delta \mathrm{F} / \mathrm{F}$ methods. Because ARES provides subcellular information about the calcium dynamics, it opens new avenues in high-resolution imaging using calcium indicators. 


\section{Materials and methods}

\section{Experimental methods}

All animal procedures were approved by the Radboud University Animal Experiment Committee. Cultures of dissociated cortical neurons were prepared from postnatal day 0 mice on C57BL/6J background. The mother was sacrificed by cervical dislocation, pups by decapitation before their brains were rapidly removed.

Preparation of neocortical dissociated cultures.

Brains were put on ice-cold Hank's balanced salts solution (HBBS; pH 7.3) comprising $10 \%$ HBSS without magnesium or calcium (Gibco Life Technologies; Catalog number (Cat.nr.): 14175129), 100 U/ml penicillin-streptomycin (Thermo Fisher Scientific; Cat.nr. 15140122) and $500 \mu \mathrm{M}$ GlutaMAX ${ }^{\mathrm{TM}}$ (Thermo Fisher Scientific; Cat.nr. 35050061). Neocortex was removed bilaterally and incubated in HBSS with $5 \%$ trypsin solution (Gibco Life Technologies; Cat.nr. 15090046). The solution was removed and the cells were dissociated using a fire polished Pasteur's pipette. Dissociated neurons were plated onto $25 \mathrm{~mm}$ coverslips, coated with $1 \%$ (weight/volume) poly-L-lysine (Sigma-Aldrich; Cat.nr. 25988-63-0), with a density of $1.6 \times 10^{5} /$ coverslip. Cultures were maintained at $37^{\circ} \mathrm{C}$ in culture medium comprising neurobasal medium (Thermo Fisher Scientific; Catalog number: 21103049) and oxygenated (95\%O2/5\% CO2), supplemented with 2\% B27 Supplement (Thermo Fisher Scientific; Cat.nr. 17504044) and $500 \mu \mathrm{M}$ GlutaMAX ${ }^{\mathrm{TM}}$ (Thermo Fisher Scientific; Cat.nr. 35050061). On day 5, the culture medium was replaced with a medium without glutamate. Half the medium was changed twice per week.

Genetically encoded calcium indicator and viral vector production.

For visualization of the intracellular calcium dynamics, we virally expressed GCaMP6s 9 under CaMK2 promoter. Viral packaging was adapted from 10 12]. In

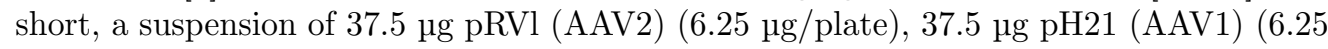

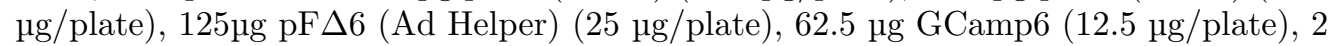
ml CaCl 2 (2.5 M; Merck; Catalog Cat.nr. 1023820500) and $12 \mathrm{ml}$ of $\mathrm{ddH}_{2} \mathrm{O}$ were slowly added ( $5 \mathrm{ml} / 15 \mathrm{~cm}$ plate) to HEK293 cells (density: 800.000 cells/plate) as plates were gently swirled. Viral transfection was confirmed 48 hours later with the Eclipse TS100 light microscope (Nikon) coupled with the epi-fluorescence illuminator Nikon Intensilight C-HGFIE and viral particles were purified with heparin (GE Healthcare; Cat.nr. 170406013). Concentration of the virus was checked with qPCR (fwd ITR primer, 5'-GGAACCCCTAGTGATGGAGTT-3', and rev ITR primer, 5'-CGGCCTCAGTGAGCGA-3'), as in 14. $2 \mathrm{\mu l}$ of the virus was transferred to dissociated neuronal cell cultures (plate diameter: $25 \mathrm{~mm}$ ) on day 2 of the culture (DIV2). For these experiments, two batches of the virus with titers of $4.92 \times 10^{12}$ (batch 6 ) and $1.86 \times 10^{12}$ (batch 7) were used. Transfected neurons were stored in the cell incubator at $37^{\circ} \mathrm{C}$ until the fluorescence was visible.

Calcium Imaging.

Neurons were visualized using LED illumination (Coolled, pE-100) and an exi blue fluorescence microscopy camera (Q-imaging, Model number: EXI-BLU-R-F-M-14-C, CA) coupled to a light microscope (Nikon, FN1) placed on an active vibration isolation table (Table Stable; TS-150). The data was acquired at full field at $10 \mathrm{fps}$ in

MicroManager (https://micro-manager.org/) at 14-bit with a readout time of $30 \mathrm{MHz}$. Data acquisition was triggered using a custom Arduino interface, coupling the whole-cell recording (see below) software Patchmaster (HEKA) with MicroManager, the program controlling the camera and the excitation light source. Electrical recordings, electrical 
stimulation and calcium imaging were time aligned with clock signals generated in the Patchmaster.

Whole cell intracellular recording and stimulation.

Cell cultures were used 9-15 days after plating, i.e. 7-13 days after viral transfection. Pyramidal neurons were visually selected according to their somatic shape and dendritic morphology. The culture plate was perfused, for the duration of the experiments, with Ringer's solutions (in mM): 10 HEPES (Sigma-Aldrich, Cat.nr. 7364459); $150.1 \mathrm{NaCl} ; 5$ $\mathrm{KCl} ; 1.5 \mathrm{CaCl}_{2} .2 \mathrm{H}_{2} \mathrm{O} ; 1 \mathrm{MgCl}_{2} .6 \mathrm{H}_{2} \mathrm{O} ; 10$ Glucose. $\mathrm{H}_{2} 0 ; \mathrm{pH} 7.4$ adjusted with $\mathrm{NaOH}$ (all last chemicals are from Merck, Catalog numbers, respectively are: 7647145, 7447407, $100350408,1058330250,14431437)$. Patch pipette electrodes with a resistance of 5-9 MOhm were pulled from borosilicate glass (Multi Channel Systems; Cat.nr. 300034) using a horizontal puller (Sutter instrument CO. Model P-2000). Intra-pipette solution included (in mM): $5 \mathrm{KCl}$ (Merck, Cat.nr. 7447407); $130 \mathrm{~K}$-Gluconate; $1.5 \mathrm{MgCl}_{2} .6 \mathrm{H}_{2} \mathrm{O}$; 0.4 Na3GTP; 4 Na2ATP ; 10 HEPES; 10 Na-phosphocreatine; 0.6 EGTA

(Sigma-Aldrich, Catalog numbers, respectively are: 299274, 1058330250, G877, A26209, 7364459, P7936, 67425). pH was set to 7.22 using KOH. (1 M; Merck; Cat.nr. 5033). A chlorided silver wire was used to create electrical continuity between the intra-pipette solution and head-stage, connected to EPC 9 amplifier (HEKA). Somatic whole-cell configuration was achieved as described before 15. Current-clamp recordings were performed using step-and-hold pulses, $500 \mathrm{~ms}$ in duration. 10 steps of $40 \mathrm{pA}$ current were delivered in every train, and each train was repeated three times. Sweep duration was set to 7 sec. Resting membrane potential was clamped at $-70 \mathrm{mV}$. Evoked calcium dynamics were visualized as described above after binning (x2) with an exposure duration of $100 \mathrm{~ms}$.

\section{Method Description}

The main algorithm uses a sliding time window over the frames composing the film, for each pixel. In the following, the time series of a single pixel will be denoted with $P$, and the value of $P$ at time (frame) $t$ will be denoted with $P_{t}$. The total number of frames (and total duration of the time series) is $T$. We compute a linear autoregression of order $k$ on the single pixel time series $P$. Calling the $k$ parameters of the linear model $U_{i}$, and the residuals $\epsilon$, we have $T$ equations and $k$ parameters (one equation with $k$ parameters for each time point):

$$
P=\sum_{i=1}^{T}\left(U_{i} P_{t-i}\right)+\epsilon_{t}
$$

For $k<T$, there are more equations than parameters: this is an overdetermined system and it can not be solved exactly. To find the set of parameters $U=U_{1}, \ldots, U_{k}$ that minimizes the residuals, we use a simple linear least-squares method [15]. Letting $P$ be a $(T \times k)$ matrix, having the $i$-lagged time series $P_{t-i}$ as the $i$-th column, $U$ the vector of the $k$ model coefficients $(k \times 1)$ and $E$ the vector of the residuals $(T \times 1)$, we can rewrite the system in matrix multiplication form: $P^{*}=\bar{P} U+E$. To find the set $\bar{U}$ that satisfies

$$
\bar{U}=\operatorname{argmin}\left(\sum_{t=1}^{T}\left|P_{t}^{*}-\sum_{i-1}^{k} \bar{P}_{t-1} U_{i}\right|\right)=\operatorname{argmin}\left\|P^{*}-\bar{P} U\right\|^{2}
$$

With the assumption that the columns of $\bar{P}$ are linearly independent, $\bar{U}$ can be computed as:

$$
\bar{U}=\left(\bar{P}^{T} \bar{P}\right)^{-1} \bar{P}^{T} P^{*}
$$


which is solvable by QR decomposition. Having found the coefficients of the linear regression $\bar{U}$, we construct the time series of the residuals $E(T \times 1)$ by comparing the measured pixel time series with the one predicted by the model. Finally, we compute the average over time of the residuals time series $E$, which constitutes the value of a single pixel in an ARES frame:

$$
A R E S=<E>=\sum_{t=1}^{T} \epsilon_{t} / T
$$

The residuals can be interpreted as noise or unpredictable (novel) events. Thanks to averaging, the noise tends to vanish, especially with longer time windows, while any transient increase or decrease in the signal is still detected. Another way to interpret the average residual time series, is to consider that we are trying to use a certain time window (of size $T$ ) of the signal to predict its future, using a linear model. The less the future time points can be predicted, the more the residuals will differ from zero.

\section{Results}

\section{Performance of ARES relative to $\Delta \mathrm{F} / \mathrm{F}$}

We introduced ARES, a new method for the analysis of functional calcium imaging data that does not require any baseline value and therefore is stimulus independent. The method proposed is based on a linear prediction of the signal by using its past (autoregression): the prediction error indicating the presence of significant variations, and therefore of an informative signal. This is especially true as calcium signals, as most biological diffusion phenomena, are essentially exponential increases, followed by exponential decays. To avoid considering the error in predicting noise as part of the resulting signal, the prediction errors are averaged over a short temporal window, which contributes to the 'lag' between ARES and raw or $\triangle \mathrm{F} / \mathrm{F}$, and how this lag decreases with increasing the intensity of the calcium response of a cell. (Fig 1 A, C). By using the past of a pixel's time series to predict its future, we take advantage of the time dimension of the data, including information of the known properties of calcium dynamics (exponential rise and decay), resulting in a better signal to noise ratio (SNR), an improved granularity, and an increased sharpness (amplitude/duration of the signal) respect to the standard ratiometric normalization $\Delta \mathrm{F} / \mathrm{F}$ methods (Fig $1 \mathrm{~B}, \mathrm{C}$ ).

\section{Effects of process order and time window}

ARES has two main hyper-parameters that need to be determined before the analysis: the order of the autoregressive process used in the autoregression (process order), and the length of the time window on which the analysis is performed (see Method description for details). The first (process order) influences the sensitivity of ARES to shorter or faster dynamics: a smaller process order corresponds to an increased sensibility to shorter transients (Fig 2). The latter, that is the number of consecutive frames taken into consideration in each autoregression (size of the time window), has a similar effect as the process order (Fig 3). Varying the window size has a linear effect on the signal: shortening the time window results in sharper signal and improved SNR, but choosing time windows that are too short $(<10$ frames recorded at $10 \mathrm{~Hz})$ does not 

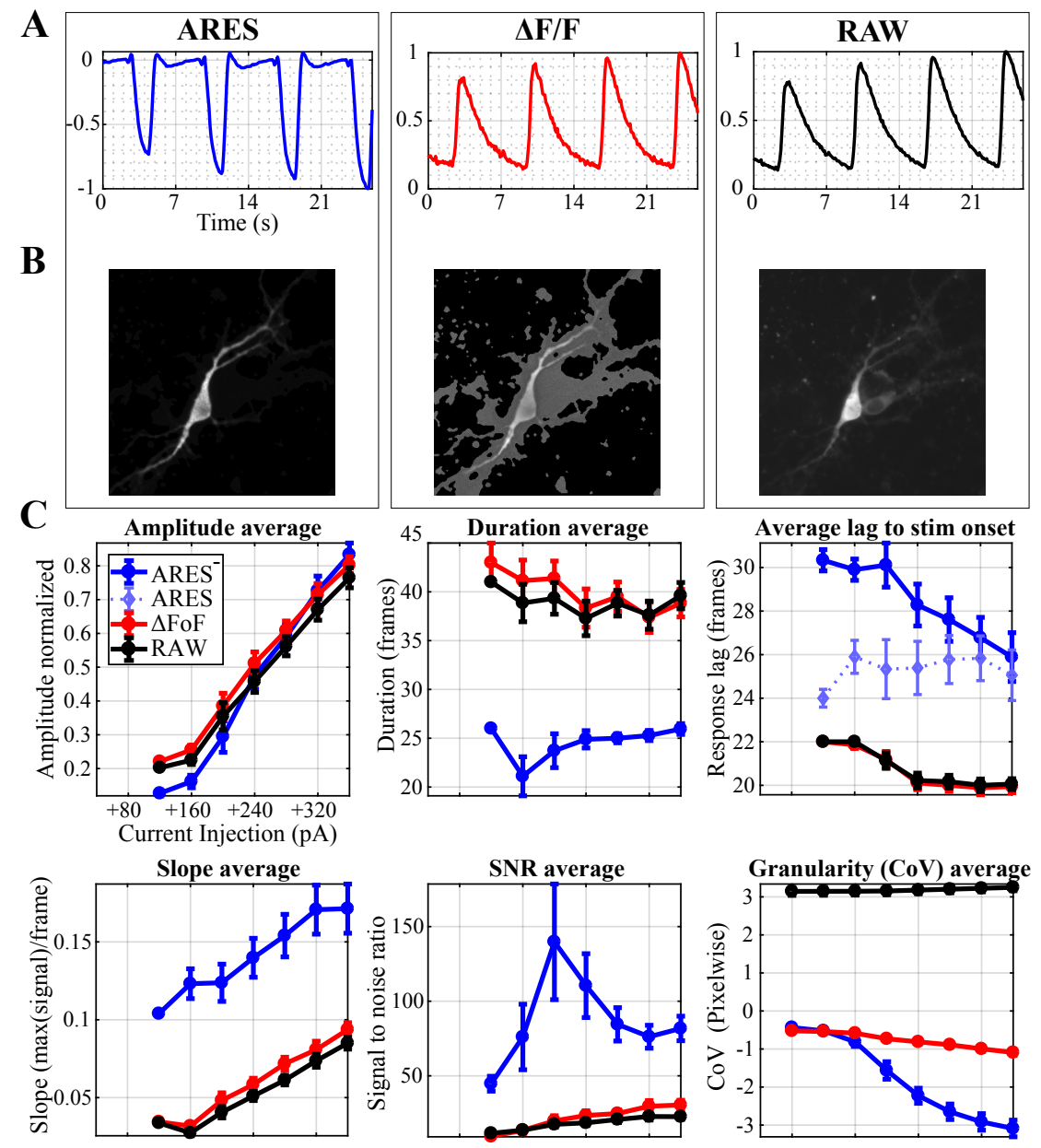

Fig 1. ARES signal and comparison with $\triangle F / F$ and raw signals. (A) A current pulse (duration: $500 \mathrm{~ms}$; intensity: increasing with increments of $+40 \mathrm{pA}$ ) was injected every 7 seconds. The traces displayed are the average projection on the time axis of each calcium response over the whole region of interest (ROI). Each trace is normalized to the global max in the time series for direct comparisons. (B) Average projection of the absolute value of each signal over the whole time lapse in the ROI. (C) Comparison between ARES, $\Delta \mathrm{F} / \mathrm{F}$ and the raw signal. From top-left to bottom right: 1) the lag between a detectable response in the signal and the stimulus onset ( 1 frame $=$ 0.1 seconds). 2) The amplitude of each trace, normalize to the maximum signal reached for comparison. 3) The duration of the response to the stimuli provided. 4) The rising slope of the signals. 5) The signal to noise ratio (noise computed by taking a sample of 100 pixels outside the cell soma and dendrites). 6) The coefficient of variation (CoV, measure of granularity of the signal, more negative is a more granular signal). Data shown are the averages and standard errors over 6 cells recordings. Each cell value is, in turn, the average over 3 repetitions of the same stimulation protocol.

allow capturing the entire duration of the calcium transient, hence ultimately reduces the SNR. The parameters can be easily adjusted with the open-source toolbox provided herein. 

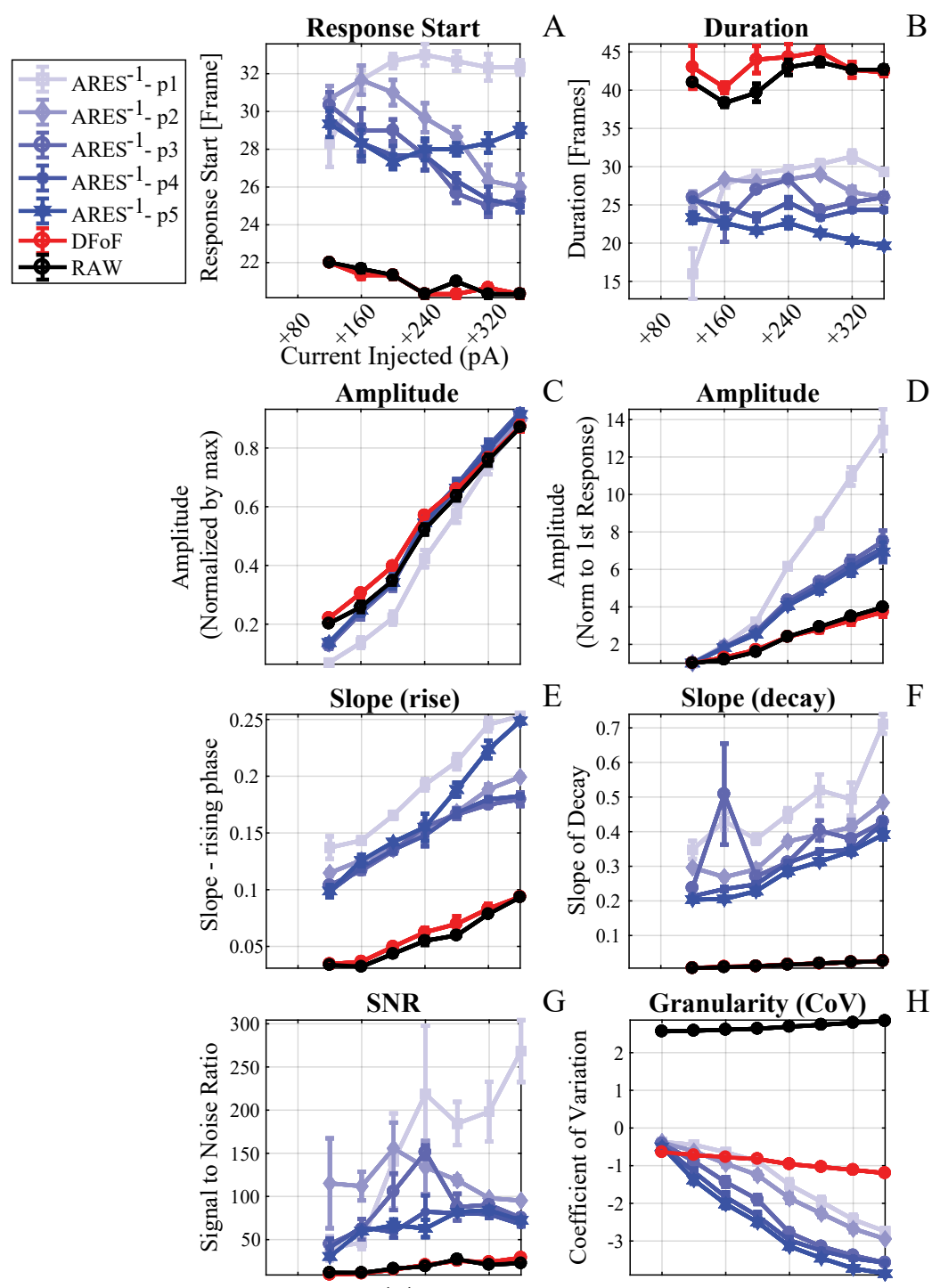

Fig 2. Effects of process order $(p)$. The order of the autoregressive process used to predict the signal is a fundamental parameter for the ARES computation. Here we showed how its values influence notable characteristics of the output, depending on the stimulation (and therefore response) level, and compare with the raw signal and $\Delta \mathrm{F} / \mathrm{F}$. In general, with the exception of the lower possible process order of 1 , the results are quite stable and comparable, most notably, the amplitude of the response $(\mathrm{C})$ both from ARES and $\triangle \mathrm{F} / \mathrm{F}$ are the same across all stimulus levels. (A) The number of frames between the stimulus and a detectable response in the signal. (B) The duration in frames of the calcium response. (C) The amplitude of the signal, normalized to the absolute maximum calcium level. (D) The amplitude of the signal normalized to the very first detectable response of the stimuli series. (E) The slope of the rising phase of the signal. (F) The slope of the decay phase. (G) The signal to noise ratio (SNR). (H) The granularity measure of the image. The process order we used by default in other figures was 3 .

\section{Tracking wave fronts}



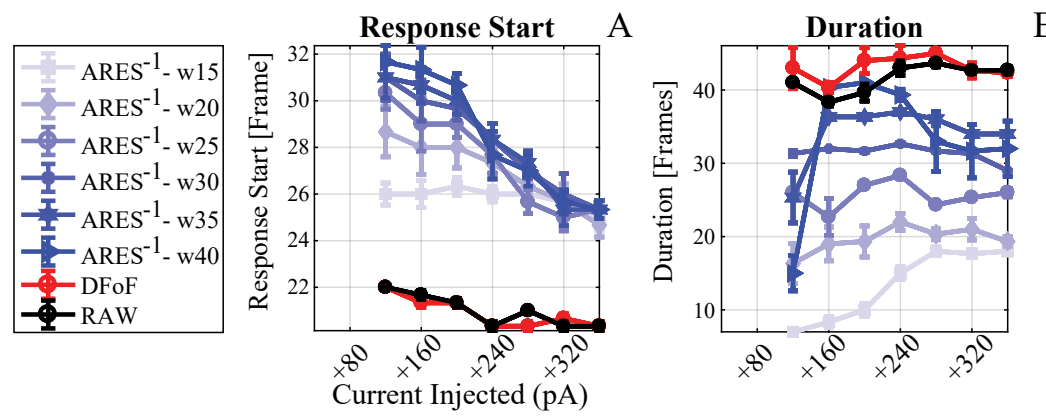

B

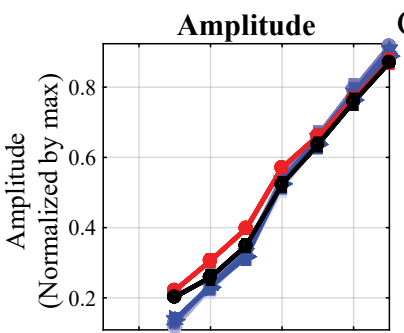

$\mathrm{C}$
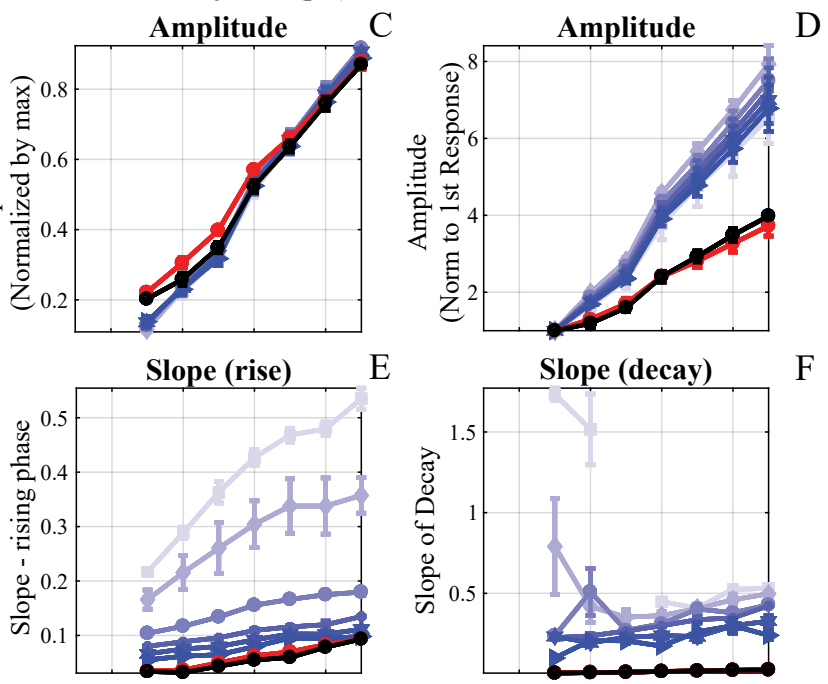

E

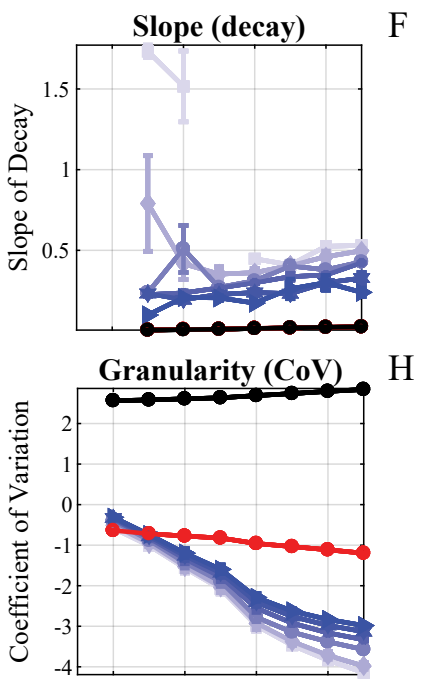

Fig 3. Effects of window size $(w)$. The length of the time window considered for the signal prediction is a fundamental parameter for the ARES computation. Here we showed how its length influence notable characteristics of the output, depending on the stimulation level. In general, the effect of varying the window size is mostly linear, with the exception of the amplitude of the response $(\mathrm{C})$ which remains stable across all stimulus levels. (A) The number of frames between the stimulus and a detectable response in the signal. (B) The duration in frames of the calcium response. (C) The amplitude of the signal, normalized to the absolute maximum calcium level. (D) The amplitude of the signal normalized to the very first detectable response of the stimuli series. (E) The slope of the rising phase of the signal. (F) The slope of the decay phase. (G) The signal to noise ratio (SNR). (H) The granularity measure of the image. The window length we used in our other examples by default in our figures was 25 frames.

the algorithm for tracking calcium wave fronts. In-vitro bright field calcium imaging data that was collected in primary neuron cultures as described (see Methods section: experimental methods). 
ARES presents an increased SNR and at the same time a signal that maintains the same amplitude of the calcium signal and the $\Delta \mathrm{F} / \mathrm{F}$ response, but without a very long tail decay (Fig 1). We show how this allows us to observe and measure the intracellular calcium influx in the form of a propagating wave coming from a dendritic spine (Fig 4 . Supplemental Video 1). We show how in the same recordings, ratiometric $\Delta \mathrm{F} / \mathrm{F}$ is still able to detect a variation in the signal at the level of the spine, but it is not able to resolve how the propagation of the wave front along the dendrite.

Because ARES numerically tracks the information content of the signal, it allows visualization and quantification of the calcium movement in subcellular resolution (Fig 4, Supplemental Video 1).

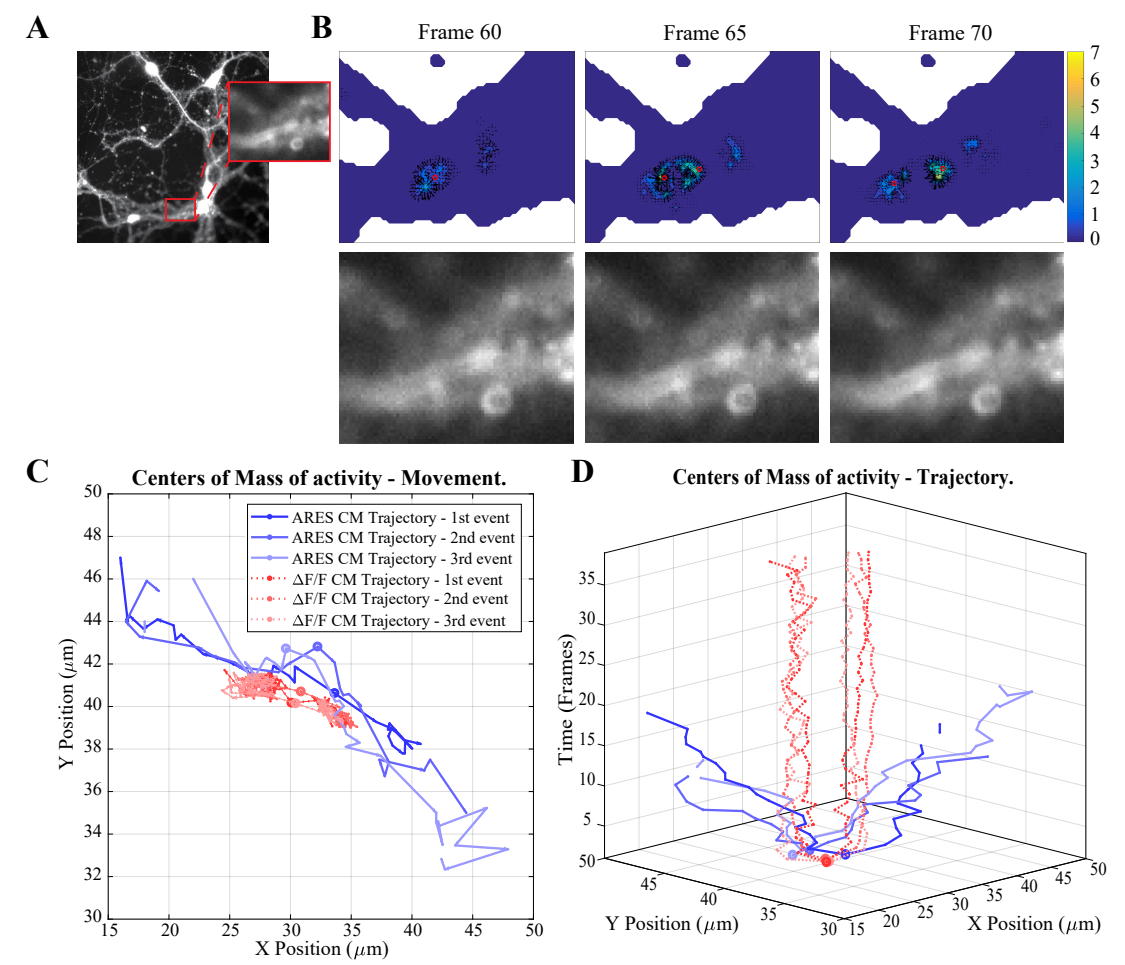

Fig 4. Spatial analysis of subcellular calcium dynamics with ARES. (A) The ROI in a dendritic spine. (B) The propagation of a calcium wave in consecutive frames (see Supplemental Film 2). Top row: the color code represents ARES values. The red circles indicate the position of the center of mass of the activity (the wavefront).

Bottom row: corresponding images analyzed with $\Delta \mathrm{F} / \mathrm{F}$. (C) The 2-D trajectory of the wave fronts, tracked as the position of their center of mass, visualized together with the time axis. Different shading of the same color represent different events recorded from the same spine. In comparison to ARES, $\Delta \mathrm{F} / \mathrm{F}$ (red) is unable to pick up any dynamic of the wave propagation.

\section{Discussion}

Ratiometric methods such as $\Delta \mathrm{F} / \mathrm{F}$ are intrinsically limited by the necessity of comparison with a single baseline value. We herein introduced a method, called ARES, that extrapolates the temporal dynamics of the signal in a pixel-wise fashion, and uses 
this knowledge to detect transient events. We showed that the method improves the temporal and spatial resolution of calcium imaging signals, while maintaining the same linearity properties as $\Delta \mathrm{F} / \mathrm{F}(\mathrm{Fig} / \mathrm{C})$. As an example application, we showed that ARES allows for the study of intracellular calcium dynamics, in the form of wave propagation from spines into dendrites.

The ARES signal is characterized by small positive increments in the signal, representing sudden rises in the calcium signal, and by larger negative peaks, representing the beginning of the signal decay. Once the decay has become linearly predictable, ARES takes zero value as there is no longer any difference between the predicted and actual signal trace. The linear regressions performed by ARES do not represent the signal dynamics by exponential rises or decays, but in doing so, the non-linear dynamics are captured in the residuals. In considering the residuals themselves, instead of the regression coefficients, anything that is not fitted by the linear regression (including the calcium transients that we are interested in) is carried by the residuals.

The improved signal resolution and transient detection of ARES, together with its baseline independence and time locality, make the method ideal for the detection of small and non-stimulus locked events, and for studying their dynamic properties. Furthermore, the toolbox accompanying the method provides an intuitive user interface, where the regression parameters can be optimized to the specific signal or indicator. The super-resolution imaging of signal transients might offer other interesting advantaged such as the differentiation between intra- and extra- cellular calcium sources, and a better reconstruction of the spiking activity, which remains a major challenge in the field. 4,5].

\section{Supporting information}

\section{Supplemental Video 1 ARES allows the tracking of a dendritic calcium} wave front The video shows imaging of a dendrite taken from primary neuronal cultures (see Material and Methods) at $10 \mathrm{~Hz}$. Four videos are presented side by side for comparison: the raw signal, a relative heatmap, and the heatmaps of $\Delta \mathrm{F} / \mathrm{F}$ and the positive part of the ARES analyzed signal, with tracking of the wave front displayed as a small red dot.

\section{Acknowledgments}

We thank Dr.Eric Jansen for help with the cell culture preparation, and the members of the Department of Neurophysiology for stimulating discussions. This work was supported by the grants from the European Commission (Horizon2020, nr. 660328), European Regional Development Fund (MIND, nr. 122035) and the Netherlands Organisation for Scientific Research (NWO-ALW Open Competition, nr. 824.14.022) to TC, the Netherlands Organisation for Scientific Research (NWO-Veni, nr. 863.150.25) to FZ, and a doctoral fellowship by the National Council for Scientific and Technological Development of Brazil (CNPQ) to ASL.

210 211 212 213 214 215 216 217 


\section{References}

1. St-Pierre F, Chavarha M, Lin MZ. Designs and sensing mechanisms of genetically encoded fluorescent voltage indicators. Current Opinion in Chemical Biology.2015;27:31-38. doi:10.1016/j.cbpa.2015.05.003.

2. Akemann W, Mutoh H, Perron A, Park YK, Iwamoto Y, Knöpfel T. Imaging neural circuit dynamics with a voltage-sensitive fluorescent protein. Journal of Neurophysiology. 2012;108(8):2323-2337. doi:10.1152/jn.00452.2012.

3. Grienberger $\mathrm{C}$, Konnerth A. Imaging calcium in neurons. Neuron.2012;73(5):862-885. doi:10.1016/j.neuron.2012.02.011.

4. Greenberg DS, Houweling AR, Kerr JND. Population imaging of ongoing neuronal activity in the visual cortex of awake rats. Nature Neuroscience.2008;11(7):749-751. doi:10.1038/nn.2140.

5. Theis L, Berens P, Froudarakis E, Reimer J, Román Rosón M, Baden T, et al. Benchmarking spike rate inference in population calcium imaging. Neuron.2016;90(3):471-482. doi:10.1016/j.neuron.2016.04.014.

6. Akerboom J, Chen TW, Wardill TJ, Tian L, Marvin JS, Mutlu S, et al. Optimization of a GCaMP calcium indicator for neural activity imaging. The Journal of Neuroscience. 2012;32(40):13819-13840.doi:10.1523/JNEUROSCI.2601-12.2012.

7. Tsien RY, Poenie M. Fluorescence ratio imaging: a new window into intracellular ionic signaling. Trends in Biochemical Sciences. 1986; p. 450-455.

8. Jares-Erijman EA, Jovin TM. FRET imaging. Nature Biotechnology.2003;21(11):1387-1395. doi:10.1038/nbt896.

9. Takahashi A, Camacho P, Lechleiter JD, Herman B. Measurement of intracellular calcium. Physiological Reviews. 1999;79(4):1089-1125.doi:10.1152/physrev.1999.79.4.1089.

10. Celikel, T. et al. Select overexpression of homer1a in dorsal hippocampus impairs spatial working memory. Front. Neurosci. 1, 97-110 (2007).

11. Freudenberg, F., Marx, V., Seeburg, P. H., Sprengel, R. \& Celikel, T. Circuit mechanisms of GluA1-dependent spatial working memory. Hippocampus 23, 1359-1366 (2013).

12. Freudenberg, F. et al. Hippocampal GluA1 expression in Gria1-/- mice only partially restores spatial memory performance deficits. Neurobiol. Learn. Mem. 135, 83-90 (2016).

13. Aurnhammer, C. et al. Universal real-time PCR for the detection and quantification of adeno-associated virus serotype 2-derived inverted terminal repeat sequences. Hum. Gene Ther. Methods 23, 18-28 (2012).

14. Celikel, T., Szostak, V. A. \& Feldman, D. E. Modulation of spike timing by sensory deprivation during induction of cortical map plasticity. Nat. Neurosci. 7, $534-541$ (2004).

15. Poznyak AS. Estimating the parameters of autoregression processes by the method of least squares. International journal of systems science.1980;11(5):577-588. doi:10.1080/00207728008967038. 

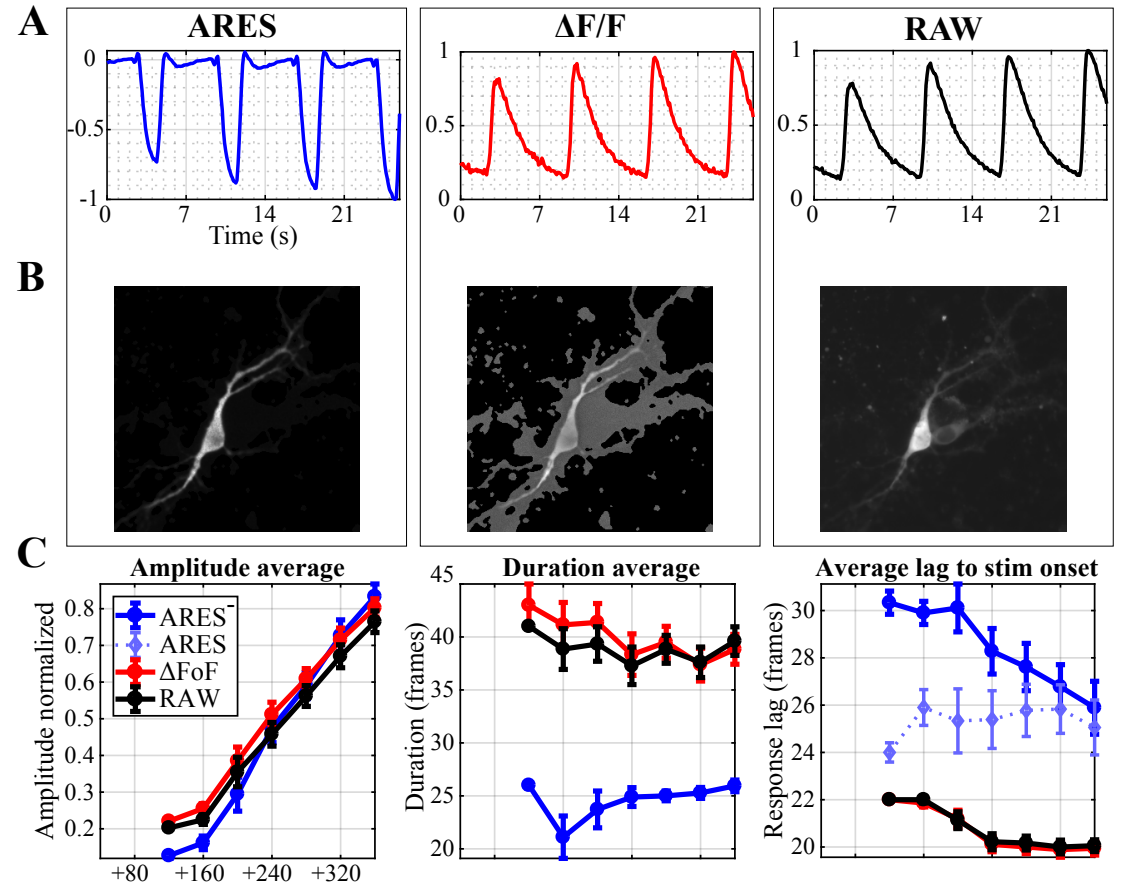

Average lag to stim onset

Current Injection (pA)
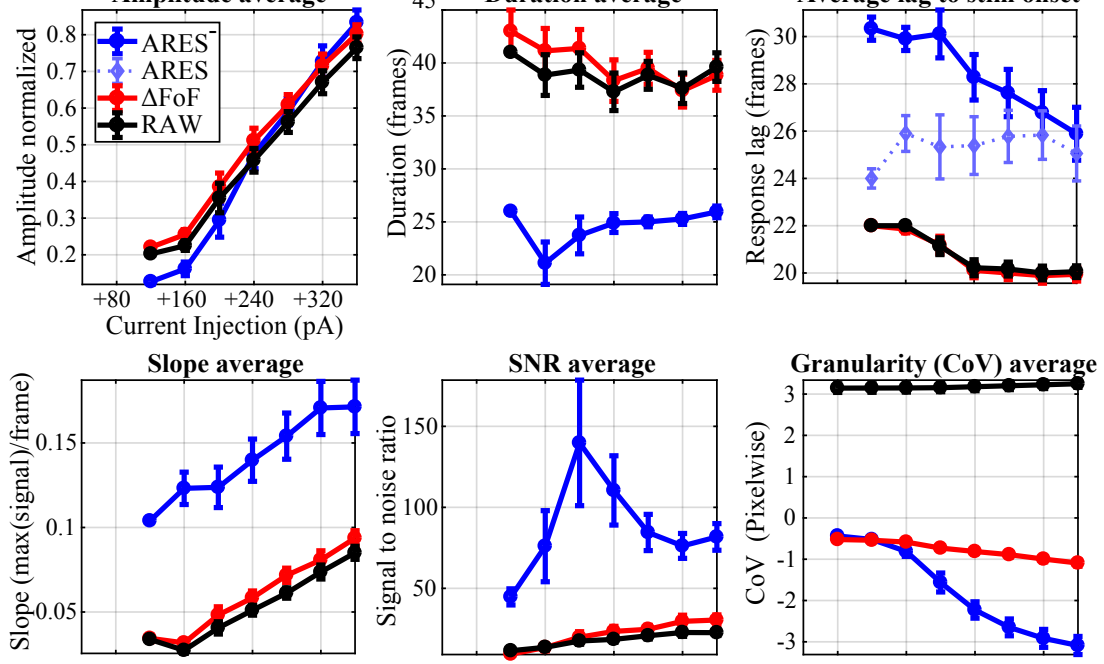


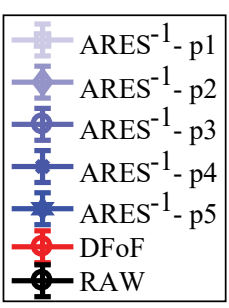

Response Start
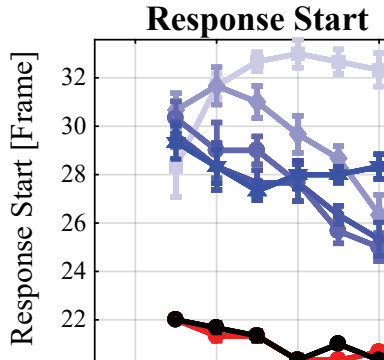

Duration

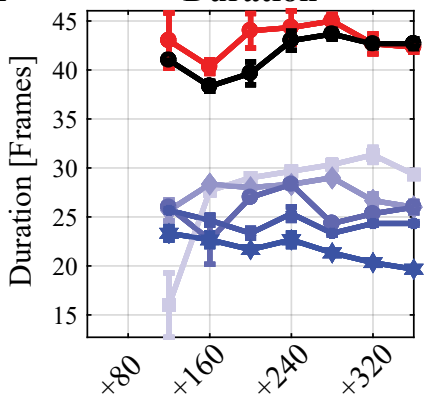

Current Injected (pA)

B

Amplitude
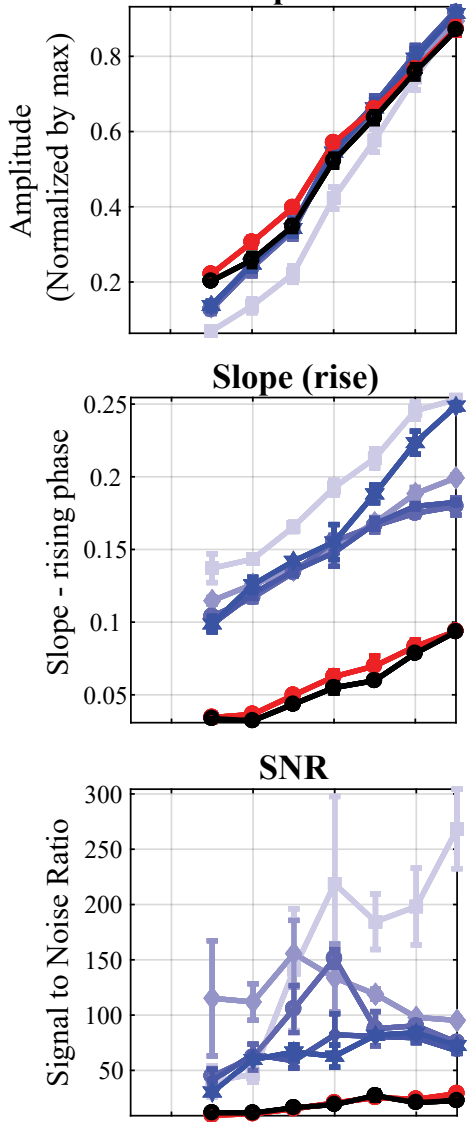

$\mathrm{C}$

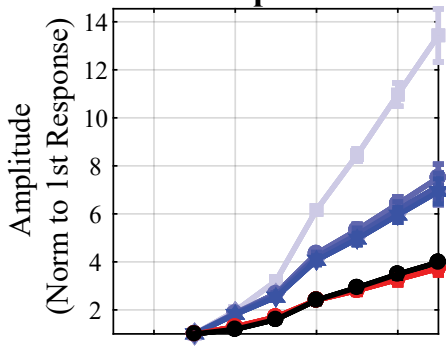

E

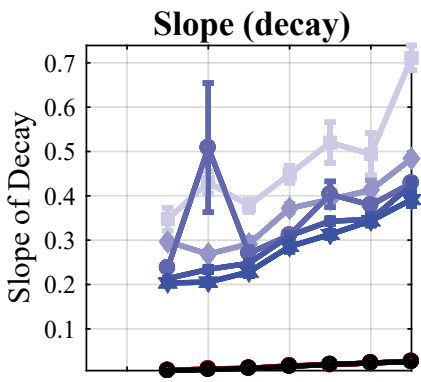

G

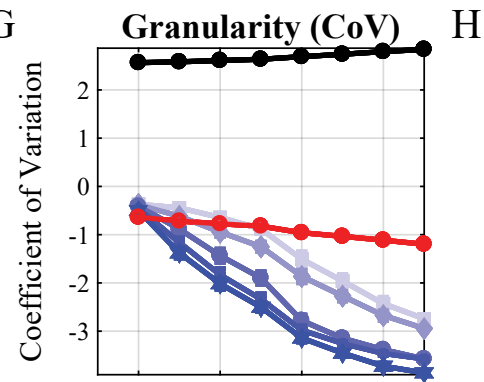


Response Start A
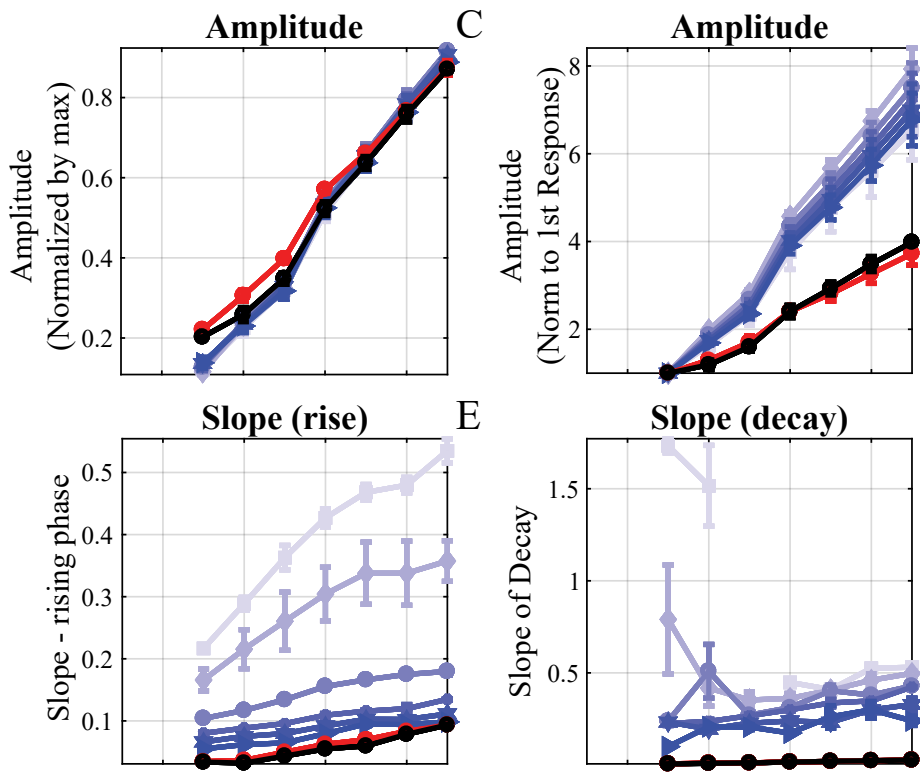

E
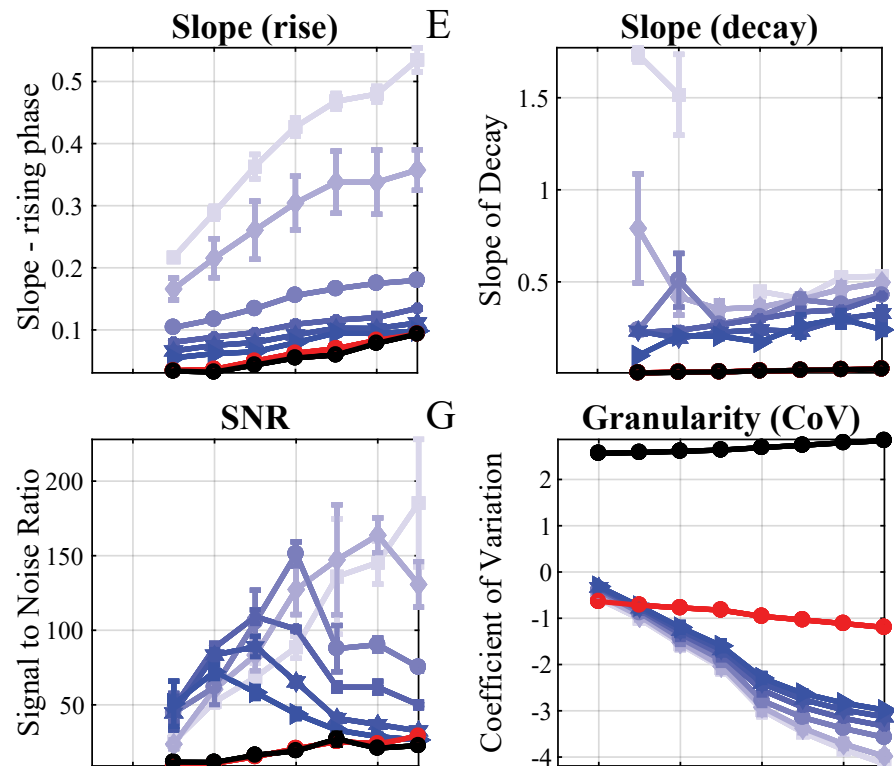

G

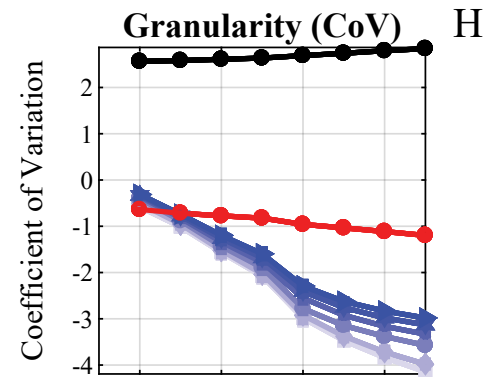


Frame 60

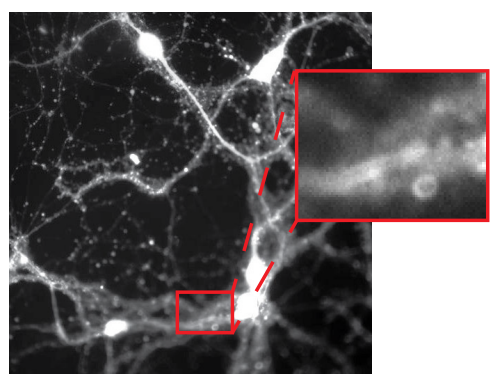

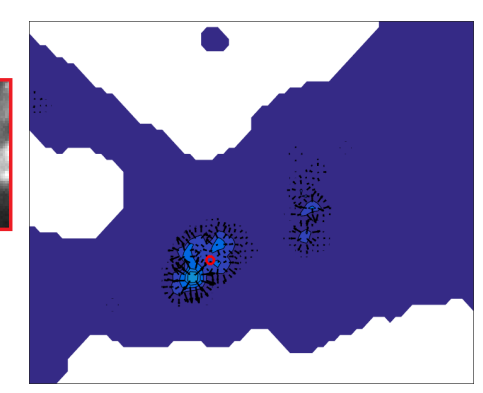

Frame 65
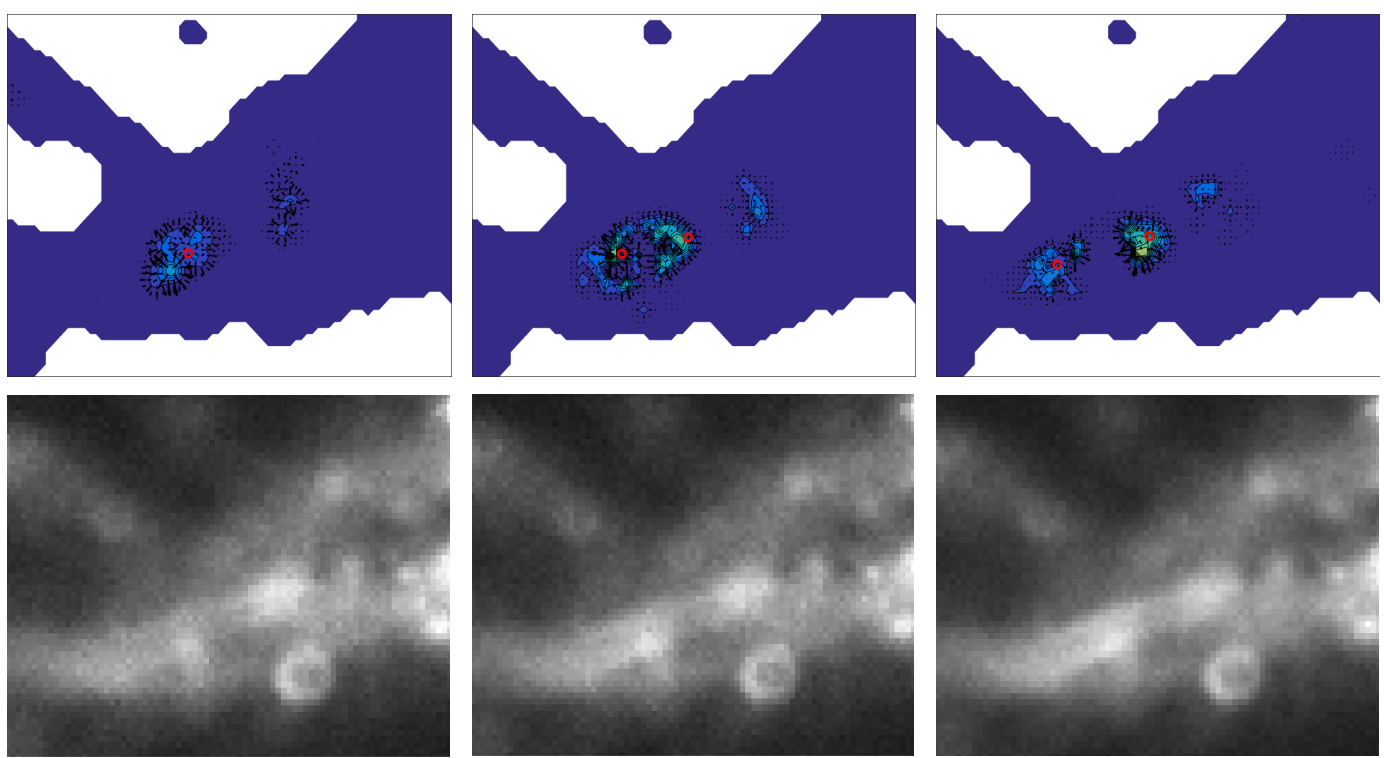

C

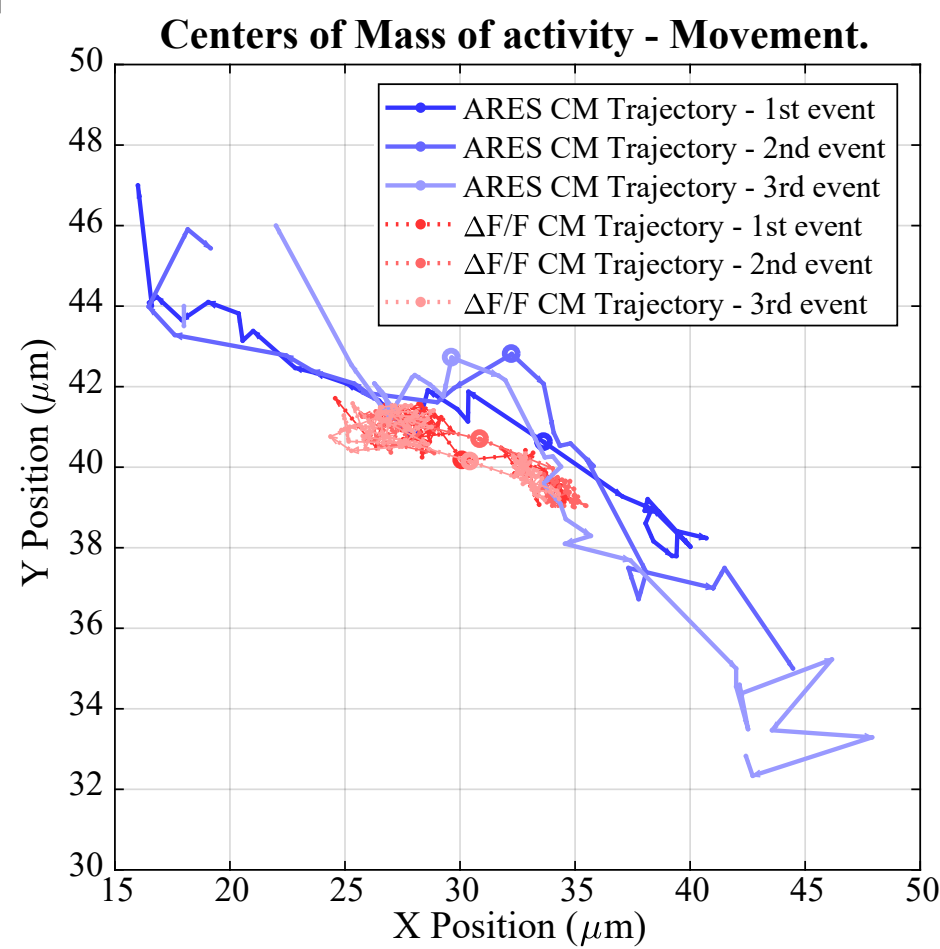

D

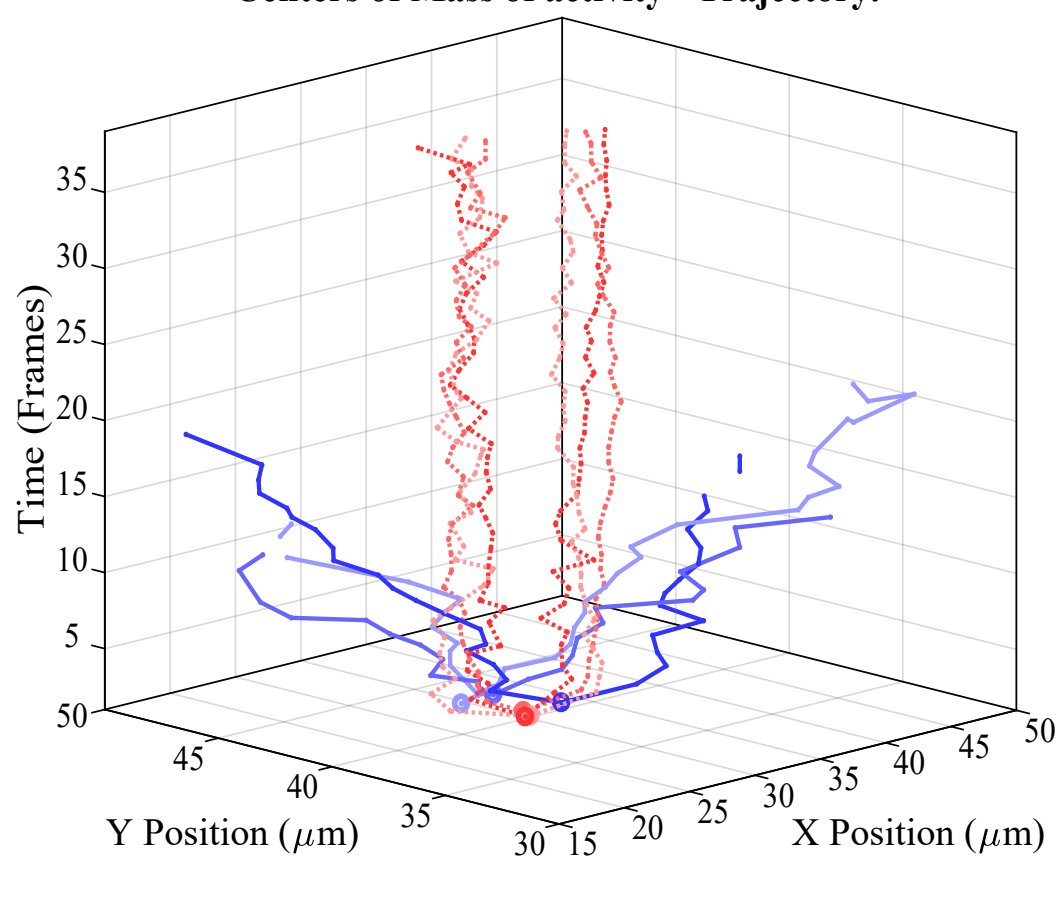

